\title{
Prevalence and antibiotic pattern of group B streptococcus among antenatal women attending a tertiary care centre in Puducherry
}

\author{
Vinod $\mathbf{R}^{1^{*}}$, Sasidharani Govindan ${ }^{2}$, Manju $\mathbf{M}^{3}$ \\ ${ }^{1}$ Associate Professor, ${ }^{2}$ CRRI, Dept. of Microbiology, Sri Venkateshwaraa Medical College \& Research Centre, Puducherry, \\ ${ }^{3}$ Associate Professor, Dept. of Biochemistry, Aarupadai Veedu Medical College \& Hospital, Puducherry, India
}

*Corresponding Author: Vinod R

Email: drvrnair@gmail.com

Received: $27^{\text {th }}$ September, 2018

Accepted: $29^{\text {th }}$ September, 2018

\begin{abstract}
Introduction: Colonization with Streptococcus agalactiae or group B Streptococcus (GBS) among antenatal mothers has become an important element which can affect the prognosis of meningitis and sepsis in neonates. Pregnant women with risk factors like preterm labour $<37$ weeks of gestation, prior GBS bacteriuria, make the situation even more vulnerable and worse.

Aims and Objectives: To find out the prevalence of group B Streptococcus (GBS) among pregnant women of Indian origin in and around our health care centre and to know the antibiotic susceptibility pattern of GBS in our region.

Materials and Methods: Rectal and vaginal swabs were collected from 126 antenatal subjects. They were cultured on to enrichment and enriched media. Group B Streptococcus (GBS) was identified by standard methods and confirmed by Latex agglutination method. Antibiotic Sensitivity Testing (AST) was done on all the isolates to know the pattern of susceptibility.

Results: Samples were collected from 126 pregnant women. Over all $11(8.73 \%)$ rectal and vaginal samples were positive for GBS. GBS was found to be higher in multigravida, 9 (9.7\%) out of 92 than primigravida, $2(5.8 \%)$ out of 34 . All the eleven isolates were sensitive to Penicillin, Ampicillin, Gentamycin, Vancomycin and Clindamycin. 2(18\%) and 1(9\%) of the GBS isolates showed resistance and moderate sensitivity to Erythromycin respectively. $8(73 \%)$ GBS isolates were sensitive to Erythromycin. All the 11 isolates were resistant to Chloramphenicol, Cotrimoxazole, Ciprofloxacin, Tetracycline and Ceftriaxone.

Conclusion: The exact prevalence of GBS must be investigated more to know its burden. Screening for GBS must be made mandatory during antenatal checkups. Intrapartum antibiotic prophylaxis (IAP) must be administered at the earliest if the screen is positive. Rapid diagnostic methods must be developed for identification of GBS.
\end{abstract}

Keywords: Streptococcus agalactiae, Group B Streptococcus, GBS, EOGBS.

\section{Introduction}

Colonization with Streptococcus agalactiae or group B Streptococcus (GBS) among antenatal mothers has become an important element which can affect the prognosis of meningitis and sepsis in neonates. They are also the current cause of pneumonia, neonatal death, septic abortion, chorioamnionitis, endometritis, cellulitis, puerperal sepsis, and premature rupture of membranes, among other perinatal infection. ${ }^{1-3}$ Pregnant women with risk factors like preterm labour $<37$ weeks of gestation, prior GBS bacteriuria, fever, prolonged rupture of membrane $\geq 18$ hours, and the reporting of neonatal infection during previous deliveries make the situation even more vulnerable and worse.

Among the pathogens affecting animals, GBS was more important cause of bovine mastitis till 1938, when it was recognized as a human pathogen. This used to be a major public health problem in the west. In the west, Centre for Disease Control (CDC), American College of Obstetricians and Gynaecologists and the American Academy of Pediatrics had formulated and revised the guidelines in 2010 for preventing GBS infections. These guidelines reinforce the need of universal screening for GBS at third semester of pregnancy. ${ }^{4,3} 10$ to $36 \%$ of women in the United States were asymptomatic carriers of GBS in the anogenital region and showed a vertical transmission rate of $50-65 \%$. Early Onset Neonatal group B Streptococcal (EOGBS) disease has now drastically diminished by $0.5 / 1000$ live births in the west due the adherence of guidelines devised by the superior health authorities. ${ }^{5,6}$

GBS disease in India is largely under recognized and highly ignorant. There are no definitive guidelines formulated by health departments for screening and prevention of GBS. Available data from India projects a $1.76 \%-16 \%$ colonization rate and vertical transmission rate of $53-56 \% .^{6}$ The present study was undertaken to assess the prevalence of GBS among pregnant women of Indian origin in and around our health care centre. We also hope that the outcome of the study can instigate researchers to probe more into this area and accumulate a nation wide data and help health authorities devise a screening protocol and prevent the neonatal damage due to colonization of GBS.

\section{Materials and Methods}

The present study was a cross sectional study, conducted over a period of one year. Permissions from the Scientific \& Research committee, Institutional ethical committee and the hospital authorities were obtained before the commencement of the study. All 
pregnant women with a gestational age of 35 or more weeks before pelvic examination attending the Obstetrics and Gynaecology (OB\&G) OPD of our tertiary care centre were included in the study.

An informed consent was also taken prior to the sampling. A detailed questionnaire regarding age, parity, gestational age and obstetric history was also noted. Two sterile cotton swabs, one from the lower third of vagina and another from rectal area were taken by a qualified Obstetrician. The swabs were immediately transported to the microbiology laboratory and immersed into Todd Hewitt broth under sterile precautions. The broth with the swabs was incubated aerobically at 35 to $37^{\circ} \mathrm{C}$ for $24 \mathrm{hrs}$. After 24 hours, the swabs were sub cultured onto $5 \%$ blood agar and kept for incubation with $5 \% \mathrm{CO}_{2}$ overnight.

Colonies which were pin point and having a narrow or complete hemolysis suggestive of GBS were further identified as GBS by Gram stain, catalase test, resistance to Bacitracin, PYR test, Christie, Atkins, Munch-Petersen (CAMP) test and hippurate hydrolysis. Serotyping with latex agglutination was also done to counter confirm the isolate as GBS. Streptex Rapid (Biomerieux) was the kit used and the manufacturer's guide lines were strictly followed while doing the test.

All the isolates were also subjected to Antibiotic Sensitivity Testing (AST) by Kirby Bauer disc diffusion method and interpreted as per CLSI guidelines. We used antibiotics like Penicillin G (10 IU), Ciprofloxacin (5 $\mu \mathrm{g})$, Vancomycin $(30 \mu \mathrm{g})$, Ceftriaxone $(30 \mu \mathrm{g})$, Ampicillin $(10 \mu \mathrm{g})$, Tetracycline $(30 \mu \mathrm{g})$, Clindamycin $(2 \mu \mathrm{g})$, Cotrimoxazole $(25 \mu \mathrm{g})$, Gentamicin $(10 \mu \mathrm{g})$, Chloramphenicol (30 $\mu \mathrm{g})$, Erythromycin $(15 \mu \mathrm{g})$ to find out the resistant and sensitive spectrum of GBS in our area. The results of the study were statistically analyzed with SPSS version 23 and the prevalence was expressed in percentages.

\section{Results}

Rectal and vaginal samples were collected from each of the 126 pregnant women with a gestational age of 35 or more weeks before pelvic examination attending the OB\&G OPD. Out of the 126 samples, 7 $(5.5 \%)$ rectal and $4(3.17 \%)$ vaginal samples were positive for GBS. Over all $11(8.73 \%)$ rectal and vaginal samples were positive for GBS out of 126 . Normal gastro intestinal flora or vaginal flora grew in rest of the115 samples. Our study subjects were in the age group between 16 - 40 yrs, hence the mean age was 26 years ( $\mathrm{SD} \pm 6.3)$. In our study, out of the 126,34 $(26.9 \%)$ were primigravida and 92 (73\%) were multigravida. Colonization with GBS was found to be higher in multigravida, $9(9.7 \%)$ out of 92 than primigravida, $2(5.8 \%)$ out of 34 .
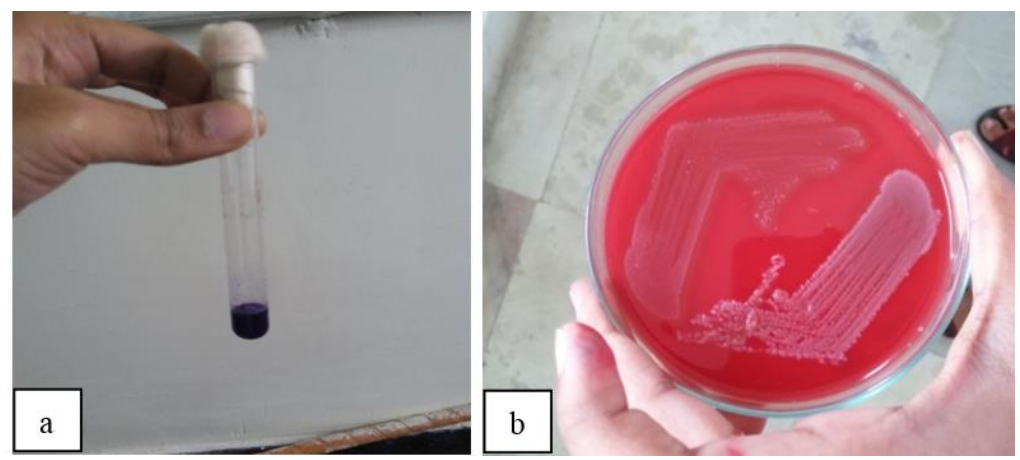

Fig. 1: a- Hippurate hydrolysis, b- GBS colonies on blood agar

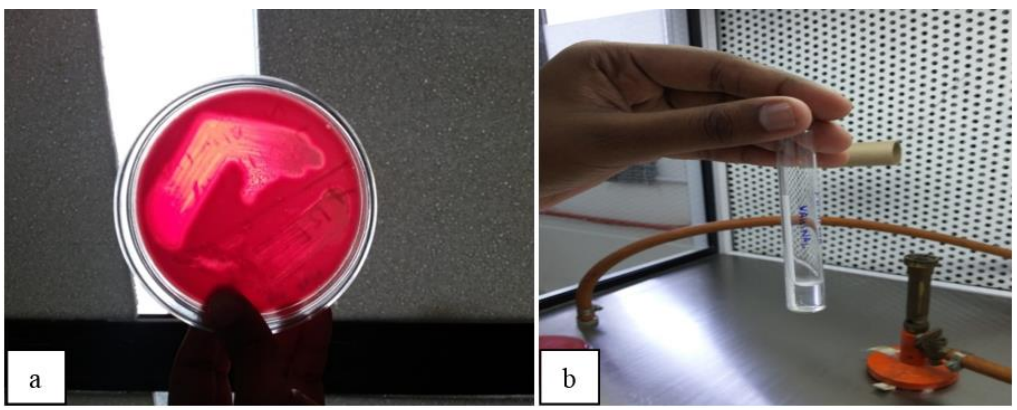

Fig 2: a - Hemolytic colonies of GBS; $b$ - Catalase test - Negative. 
Table 1: Variables compared in GBS positive \& negative women

\begin{tabular}{|l|c|c|c|}
\hline \multicolumn{1}{|c|}{ Variables } & \multicolumn{3}{|c|}{ GBS Colonization } \\
\cline { 2 - 4 } & Total n (\%) & Positive n (\%) & Negative n (\%) \\
\hline Age (yrs) & & & \\
$16-20$ & $7(5.5)$ & $0(0)$ & $7(5.6)$ \\
$21-25$ & $36(28.5)$ & $5(3.9)$ & $31(25)$ \\
$26-30$ & $50(39.6)$ & $4(3.17)$ & $46(37)$ \\
$31-35$ & $28(22.2)$ & $2(1.6)$ & $26(21)$ \\
$36-40$ & $5(3.9)$ & $0(0)$ & $5(4)$ \\
\hline Rectal colonization & & $7(5.5)$ & $119(94.4)$ \\
\hline Vaginal colonization & & $4(3.17)$ & $122(97)$ \\
\hline Primigravida & $34(26.9)$ & $2(5.8)$ & $32(25.3)$ \\
\hline Multigravida & $92(73)$ & $9(7.14)$ & $83(66)$ \\
\hline
\end{tabular}

The AST done on the isolates revealed the following pattern. All the eleven isolates were sensitive to Pencillin, Ampicillin, Gentamycin, Vancomycin and Clindamycin. 2(18\%) and $1(9 \%)$ of the GBS isolates showed resistance and moderate sensitivity to Erythromycin respectively, $8(73 \%)$ GBS isolates were sensitive to Erythromycin. Multi drug resistance was seen among Chloramphenicol, Cotrimoxazole, Ciprofloxacin, Tetracycline and Ceftriaxone.

Table 2: Antibiotic sensitivity testing (AST)

\begin{tabular}{|l|c|c|c|}
\hline \multicolumn{1}{|c|}{ Antibiotic } & Sensitive & Moderate & Resistant \\
\hline Pencillin & $11(100 \%)$ & - & - \\
\hline Ampicillin & $11(100 \%)$ & - & - \\
\hline Gentamycin & $11(100 \%)$ & - & - \\
\hline Vancomycin & $11(100 \%)$ & - & - \\
\hline Clindamycin & $11(100 \%)$ & - & - \\
\hline Erythromycin & $8(73 \%)$ & $1(9 \%)$ & $2(18 \%)$ \\
\hline Ciprofloxacin & - & - & $11(100 \%)$ \\
\hline Chloramphenicol & - & - & $11(100 \%)$ \\
\hline Cotrimoxazole & - & - & $11(100 \%)$ \\
\hline Tetracycline & - & - & $11(100 \%)$ \\
\hline Ceftriaxone & - & - & $11(100 \%)$ \\
\hline
\end{tabular}

\section{Discussion}

The burden of asymptomatic colonization of GBS and the mortality and morbidity caused by this isolate is not a seriously recognized problem globally and in our country. This is evidenced by the meagre studies available for assessment. Early recognition of colonizers can save many neonatal lives. Identifying the potential subjects and treating them with a course of sensitive antibiotics during intrapartum period can definitely curb the prevalence of GBS neonatal disease. As per a review on prevention of perinatal GBS infection, the prevalence of asymptomatically colonized antenatal women in the United States is 10-35\% and almost 1.7 cases per 1000 live births have invasive GBS infection. ${ }^{7-9}$

In the United Kingdom the incidence is 0.5 per 1000 live births. According to the available studies on prevalence of GBS in India, the colonization rate in pregnant women is $9.7 \%$ to $16 \%$ and the incidence of neonatal GBS is 0.17 per 1000 live births. ${ }^{8,10,11}$ Reports from other studies conducted globally and in southern states of India suggest variation in GBS colonization among area, origin and even the development of a country. As per their data developed countries had high prevalence rates when compared to developing countries. The data collected revealed that in UK it is $21.3 \%$, USA had a prevalence rate of $15-40 \%$ and Canada showed $19.5 \% .^{12-14}$ Comparing this, the prevalence rate in Vellore (India) is 2.6\%, Chengalpattu (India) is $8 \% .^{15,16} \mathrm{~A}$ study in Puducherry conducted during 2011 showed a prevalence of $2.3 \% .{ }^{17}$ In our study we could find the prevalence rate was $8.73 \%$, this prevalence rate is alarming when compared to other studies conducted in the southern region of India. Institution catering to antenatal mothers must conduct more number of studies to bring out the unrecognized prevalence of GBS in the pregnant population.

GBS screening is not mandatory in many health care facilities globally and even in our country. In countries like the US and UK where the universal screening for GBS was adopted and the use of Intrapartum Antibiotic Prophylaxis (IAP) made mandatory for screen positive mothers the incidence of neonatal GBS has significantly been lowered. More number of studies in our region will definitely reveal the increased burden of GBS disease. This should alarm the health authorities to make universal GBS screening mandatory.

Standard bacteriological culture methods are still the gold standard to isolate GBS. ${ }^{9}$ In our study enriching the isolate with Todd Hewitt broth could increase the rate of recovery. There is a need for developing highly sensitive and specific rapid test to detect GBS within few hours to administer IAP at the earliest. Penicillin is still the drug of choice if not allergic. In our study also all the isolated GBS were sensitive to Penicillin. Clindamycin, an alternate drug in allergic circumstances to penicillin was sensitive in our study. Globally $10 \%$ resistance has been reported against Clindamycin. ${ }^{18}$ Erythromycin resistance was observed in our study, the same was also reported in another study from Puducherry. ${ }^{17}$ The present study was also conducted in another area of Puducherry; 
hence resistance pattern to Erythromycin in other areas has to be probed. Since there are not much studies probing into the overall antibiotic pattern of GBS the exact resistance pattern is also unknown. In our study we observed an association of GBS colonization with multiparty, another study conducted in Trinidad has also reported the same. ${ }^{19}$

Multiple exposures during deliveries in health care settings and an element of health care associated infection also have to be investigated and considered as a factor for GBS colonization in multigravida. In summary health care facilities which cater antenatal and paediatric well health must consider studying the prevalence of GBS in their areas with antibiotic pattern for successful IAP. This prevalence should instil the minds of health authorities to develop mass screening program and include them in national health programs to protect any neonate with EOGBS. Rapid identification methods must be the need of hour to start IAP at the earliest.

\section{References}

1. Mônica T, Dayana F, Maria R T. Screening for group B Streptococcus in pregnant women: a systematic review and meta-analysis. Rev Latino Am Enfermagem. 2011;19(6):1470-8.

2. CDC. Prevention of perinatal Group B Streptococcal Disease. A Public Health Perpective. MMWR-Recomm Rep. 1996(RR-7):1-24.

3. CDC. Prevention of Perinatal Group B Streptococcal Disease Revised Guidelines from CDC, 2010. MMWR. 2010;59(RR-10):1-27.

4. CDC. Prevention of Perinatal group B Streptococcal disease. Revised Guidelines from CDC. MMWR Recomm Rep. 2002;51(RR-11):1-22.

5. Schrag S, Gorwitz R, Butts K F, Schuchat A Centers for Disease Control and Prevention. Prevention of perinatal group B Streptococcal disease. Morb Mortal Wkly Rep. 2002;51(11):1-22.

6. Patil K P, Singla S S, Nagamoti M B, Samy M K. Grop B Streptococci colonization in pregnant women: Is screening necessary?. J South Asian Feder Obst Gynae. 2013;5(2):64-67.

7. Shet A, Ferrieri P. Neonatal and maternal group B streptococcal infections: A comprehensive review. Indian J Med Res. 2004;120:141-50.

8. Narava S, Rajaram G, Ramadevi A, Prakash, GV, Mackenzie S. Prevention of perinatal group B streptococcal infections: A review with an Indian perspective. Indian J Med Microbiol. 2014;32:6-12.

9. CDC, Morbidity and Mortality Weekly Report (MMWR). Prevention of Perinatal Group B Streptococcal Disease. Revised Guidelines from CDC, 2010 Recommendations and Reports November. 19, 2010/59(RR10);1-32.

10. Dalal BS, Lahiri A, Parel CC. Carriage rate of group B streptococci in pregnant women and evaluation of different isolation media. $J$ Indian Med Assoc. 1998;96:360-366.

11. Chaudhary U, Sabherwal U, Chugh TD. Prevalence of group B streptococci in obstetrical cases. Indian J Med Res. 1981;73:710-4.

12. Davies HD, Adair C, McGeer A, Ma D, Robertson S, Mucenski M, et al. Antibodies to capsular polysaccharides of group B Streptococcus in pregnant
Canadian women: relationship to colonization status and infection in the neonate. J Infect Dis. 2001;184:285-91.

13. Jones N, Oliver K, Jones Y, Haines A, Crook D. Carriage of group B streptococcus in pregnant women from Oxford, UK. J Clin Pathol. 2006;59:363-6.

14. Campbell JR, Hillier SL, Krohn MA, Ferrieri P, Zaleznik DF, Baker CJ. Group B streptococcal colonization and serotype-specific immunity in pregnant women at delivery. Obstet Gynecol. 2000;96:498-503.

15. Mani V, Jadhav M, Sivadasan K, Thangavelu CP, Rachel M, Prabha J. Maternal and neonatal colonization with group B Streptococcus and neonatal outcome. Indian Pediatr. 1984;21:357-63.

16. Balan K, Abirami Lakshmy, Ashwini MA, Vijayalakshmi TS, Sheila Doris Devamani. Screening for group B Streptococci in antenatal women: Isolation and antibiogram. Indian J Microbiol Res. 2018;5(2):151-153.

17. Sharmila V, Joseph NM, Arun Babu T, Chaturvedula L, Sistla S. Genital tract group B streptococcal colonization in pregnant women: a South Indian perspective. J Infect Dev Ctries. 2011;5:5925.

18. Royal College of Obstetricians and Gynaecologists. Prevention of Early-onset Neonatal Group B Streptococcal Disease. Guideline No. 36, 2nd ed. London: RCOG; 2012.

19. Orrett FA. Colonization with Group B streptococci in pregnancy and outcome of infected neonates in Trinidad. Pediatr Int. 2003;45:319-323.

How to cite this article: Vinod R, Govindan S, Manju M. Prevalence and antibiotic pattern of group B streptococcus among antenatal women attending a tertiary care centre in Puducherry. Indian J Microbiol Res. 2018;5(4):466-469. 